\title{
Mobile eLearning and Technology (MeLT)
}

Authors: Keith Brown and Julie Letchford. University of Bath

Background: Although mobile devices are widespread, the full potential for teaching and learning has yet to be exploited. A recent survey $(n \sim 1200)$ indicates that:

- $92 \%$ of undergraduates have a mobile phone and around $36 \%$ have a tablet.

- Mobile apps are strongly desired by students. This includes apps for library services, past-papers, lectures, e-books, timetable and quizzes.

\section{Method:}

1. Identify apps desired by students

2. Build apps for phone/tablet delivery on Android and iOS platforms

3. Trial apps with undergraduates

Results: A number of 'app-store' type apps have been developed and evaluated with Pharmacy students. This includes a timetable app, campus map and apps for two first-year units - Introduction to Microbiology and Pharmaceutical Analysis. These unit-apps include lecture slides and interactive quizzes together with notetaking facilities for annotating the lecture slides. The Timetable app was released for Android in April 2013 and trialled with students ( $n \sim 67)$ from all year groups. Results indicate that it is accurate, easy-to-use, and popular with users. The app 'Introduction to Microbiology' was released for Android in November 2013 and trialled with a small number of first-year students $(n \sim 15)$. The evaluation results indicate:

- $93 \%$ find the app useful

- $93 \%$ find the app easy-to-use

- $100 \%$ would use the app for private study

Recently, a private app-store capability for both iOS and Android has been implemented. This facility will enable roll-out and evaluation to all first-year pharmacy students next semester.

Conclusion: Apps help to provide an integrated educational experience: Learning is available anytime, anywhere, even when there is no wi-fi or mobile connection. By integrating apps into the MPharm degree, it is hoped to deliver learning materials in a way that is both intuitive and effective for students. 